\title{
DETC2007-35264
}

\section{TACTILE WHOLE-FIELD IMAGING SENSOR ON PHOTOELASTICITY}

\author{
Venketesh N Dubey and Gurtej S Grewal \\ School of Design, Engineering \& Computing \\ Bournemouth University \\ Talbot Campus, Fern Barrow \\ Poole, BH12 5BB, UK \\ Tel. +441202965986 \\ Fax. +44 1202965314 \\ Email: vdubey@bmth.ac.uk
}

\begin{abstract}
The paper describes a whole-field imaging sensor developed on the principles of photoelasticity. The sensor produces colored fringe patterns when load is applied on the contacting surface. These fringes can be analyzed using conventional photoelastic techniques, however, as the loading in the present case is not conventional some new strategies need to be devised to analyze the load imprint. The loading is unconventional in the sense that low modulus photoelastic material is deformed under vertical load in the direction of light travel to induce the photoelastic effect. The paper discusses the efficacy of both RGB calibration and phase shifting techniques in sensing applications. The characteristics of fringe patterns obtained under vertical and shear loads have been studied and the results obtained under these conditions are discussed with their limitations specifically when this is applied for sensing applications. Finally a case study has been conducted to analyze a foot image and conclusions drawn from this have been presented.
\end{abstract}

Keywords: Whole-field Sensor, Photoelasticity, RGB Methods, Phase unwrapping, Unconventional Loading

\section{INTRODUCTION}

Photoelastic materials exhibit temporary birefringence when subjected to mechanical stresses. This produces colored fringes in the material under white light, which can be visualized through suitable optical filters. This characteristic is based on the phenomenon possessed by certain non-crystalline materials that are ideally isotropic but behave anisotropically when loaded [1]. The effect is temporary and persists only until specimen is loaded. This has conventionally been used for analyzing stress distribution in loaded models, however, this effect can potentially be used in sensing applications if the fringe information can be precisely quantified in terms of stress distribution. The advantage of using this technique is to get whole-field visualization of the stress field, which may provide load information of the entire field as opposed to employing strain gauges, or load cells that only offer discrete load information. This property of the material has been used in developing a number of discrete signal-based sensors [2-4], however, as the fringe patterns contain details of the loading conditions this can be exploited for developing a whole-field sensor. The same technique can be enhanced for any generalized case involving complex fringe patterns under different loading conditions for whole-field analysis. This may find application in a number of sensing areas including biomedical and robotics for example, to assess the footprint of diabetic patients or to analyze pressure patterns of disabled subjects and thereby enacting prevention strategies by suitable footwear or bed design. The technique may also be suitable for developing haptic interfaces in robotic applications or humancomputer interface [5]. This will require use of suitable photoelastic material that can have a wide dynamic range and is sensitive to different loading conditions. These requirements make the conventional photoelasticity depart from the existing stress analyzing techniques, which may not be applicable in a certain class of loading situations. For example, in order to obtain imprint of foot loading condition, low modulus material 
has to be used and loading has to be applied in the direction of light travel. This is atypical of conventional photoelasticity, where loading is applied across the light travel for pronounced photoelastic effect [6]. This paper evaluates the use of conventional photoelastic techniques under such loading conditions and discusses their limitations and proposes alternative approaches that may be necessary for sensing applications.

\section{SENSING ELEMENT AND LOADING CONDITIONS}

The conventional photoelastic techniques are generally used for surface stress problems, however, if this technique is applied for sensing applications, direction of applied load becomes an important factor which needs to be considered carefully. For pronounced photoelastic effect to take place the forces need to be applied perpendicular to the direction of light travel and thus tangential to the sensing surface. However, for forces in the direction parallel to the incident light (perpendicular to the surface), which may be required in sensing applications, some indirect method of analysis must be used (since the photoelastic effect is generated due to material deformation), such as contact stress analysis or techniques to investigate the effect of load on perpendicular planes. In the present case, photoelastic effect is induced due to vertical force on the surface of photoelastic material, which means that direct photoelastic relationships may not generate accurate result.

The arrangement of various elements of the sensing module is shown in Figure 1. A $3 \mathrm{~mm}$ photoelastic sheet was glued to a $15 \mathrm{~mm}$ thick polycarbonate sheet using clear adhesive of matching elasticity. The photoelastic material used in the experiment has a low modulus of elasticity of $4 \mathrm{MPa}$, which is suitable for dynamic or wave propagation studies [7]. This makes it ideally suited for the intended sensing application. The top surface was coated with a reflective paint with appropriate elastic properties to provide a reflective backing. This forms the sensing surface where load can be applied and fringes can be viewed from the other side i.e. through the polycarbonate sheet with suitable filters [8]. The polycarbonate sheet served as a strong support for the low modulus photoelastic sheet during load application.

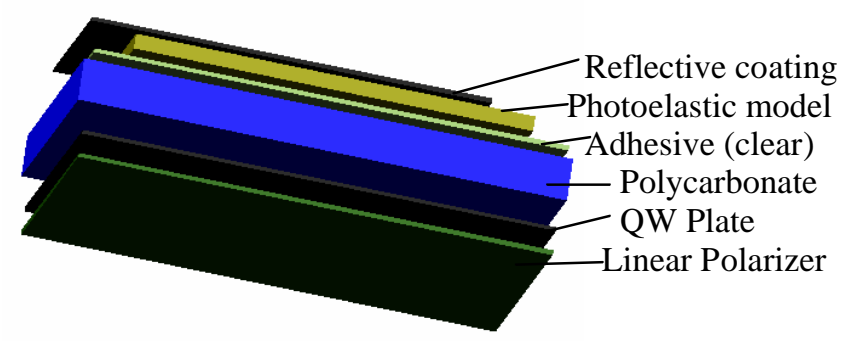

Figure 1. Elements of the sensing module

A set of indenters is designed to apply controlled vertical and shear loads to the material. Figure 2 shows the design of the indenter plate with four indenter heads. The images obtained from the sensing plate under vertical and shear force are shown in Figure 3. The figures show the fringe pattern developed when a force of $8 \mathrm{~N}$ is vertically applied by an indenter of $3.5 \mathrm{~mm}$ diameter. It can be seen that the patterns are generally symmetrical. When a shear force of $8 \mathrm{~N}$ is introduced to the vertical force the fringes are shifted (adjacent figure) in the direction of shear. It is interesting to see that the photoelastic effect is appreciable outside the point of indentation. It is this property of the photoelastic material to automatically magnify the load makes it ideally suited for such sensing applications, which can be used for measuring vertical as well as shear forces which may exist on the plane.

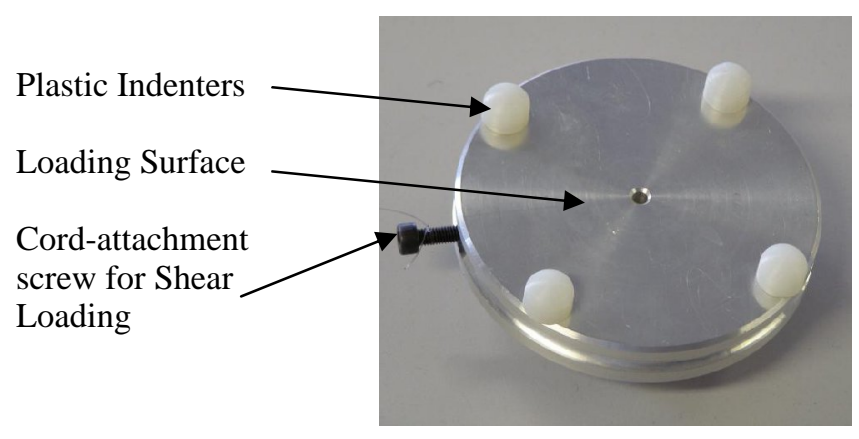

Figure 2. Loading plate with indenters

These images are representative of a whole-field sensor where the entire area covered can be mapped against the stimulating forces, however, these fringes are produced as a result of deformation of material so stress concentration at the point of application is too large. Also the photoelastic effect (colored fringes) under the indenter head is not pronounced since load is applied in the direction of the light travel. Therefore, no useful stress information can be extracted from this region.
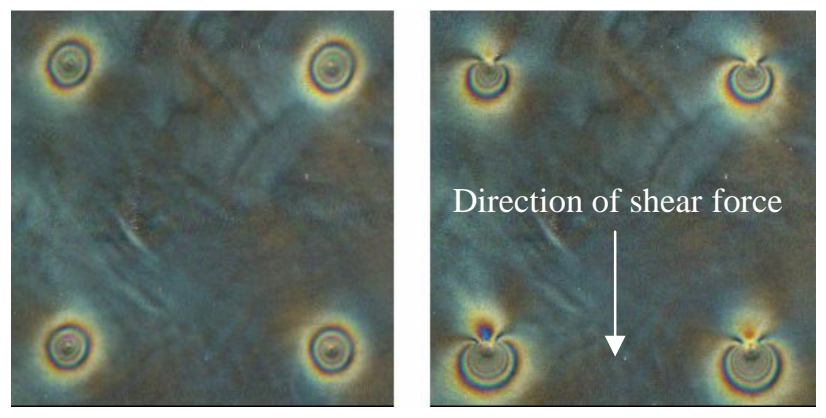

Figure 3. Fringe patterns under (a) vertical, (b) shear loads

\section{PHOTOELASTIC IMAGE ANALYZING TECHNIQUES}

The most commonly used photoelastic techniques are RGB calibration [9-11] and phase-shifting [12-14] methods. The RGB calibration is a simple technique which provides isochromatic (stress) information in terms of fringe order. The technique requires generation of a look up table (LUT) and extracting fringe order from the table for test points. Under 
white light stress information is represented in form of colored fringe patterns which appear in sequence. The technique exploits this fact to generate a LUT with color information against fringe order. During analysis the RGB triplet value of test point can be compared to the LUT values using least square method (equation 1) thus fringe order can be determined and stress analysis can be performed.

$$
e=\left(R_{s}-R_{m}\right)^{2}+\left(G_{s}-G_{m}\right)^{2}+\left(B_{s}-B_{m}\right)^{2}
$$

where $e$ is the error function, $(R G B)_{m}$ are measured RGB triplet values at the test point and $(R G B)_{s}$ are the stored RGB triplet values in the LUT. The technique is not computationally intensive, however, only fringe order up to three can be determined relatively accurately with some sparse errors. The technique is prone to errors for higher fringe orders i.e. highly stressed zones with densely packed pixels cannot be determined accurately and requires some advanced fringe tracking algorithms and special light sources [15].

Phase shifting, on the other hand, is the most promising multi-image technique providing both isoclinic (direction) and isochromatic information. It is based on acquisition of phase stepped images of photoelastic model at different orientation of optical elements. Intensity equations from these images are used for determination of isochromatics and isoclinics. An algorithm is required for phase unwrapping of the data obtained from these equations.

Equations (2-3) are used to determine isoclinics and isochromatic respectively, and are derived from the intensity equations of six phase stepped images.

$$
\begin{aligned}
& \theta=\frac{1}{2} \tan ^{-1}\left(\frac{i_{5}-i_{3}}{i_{4}-i_{6}}\right) \\
& \delta=\tan ^{-1}\left(\frac{i_{4}-i_{6}}{\cos 2 \theta\left(i_{1}-i_{2}\right)}\right)
\end{aligned}
$$

where $\theta$ is isoclinic value, $\delta$ is isochromatic value and $i_{n}$ are intensity representation of phase stepped images, $(n=1,2 \ldots 6)$. This method works well, however, errors are prominent at the point of loading and are difficult to unwrap.

\section{SENSOR DESIGN}

The experimental sensor developed to conduct LUT based calibration in RGB photoelasticity is shown in Figure 4. Due to the requirements of application, reflection photoelasticity was implemented in these experiments. This requires only one set of linear and quarter wave plate or one circular polarizer. The placing of polarizer, quarter wave plate and spacing between scanner bed and the model is slightly exaggerated in the figure, but in actual setup they form a compact unit. Load applied on the photoelastic material generates colored fringes under white light which can be recorded by a digital camera for processing.

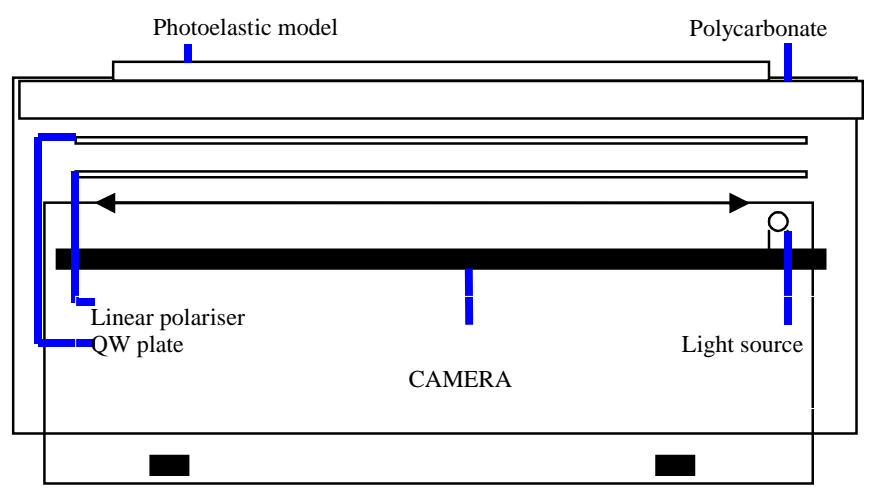

Figure 4. Sensor design for RGB calibration method

As the application is based on reflection mode, for phaseshifting experiments images at different orientations of optical elements are required, thus use of partial mirror was made. The setup uses a separate light source and a camera oriented orthogonally for the ease of operation in this experiment. Figure 5 shows a schematic diagram of the design with optical filters. Using a partial mirror gives an advantage of a portable setup at the cost of light intensity due to partial reflection and transmission.

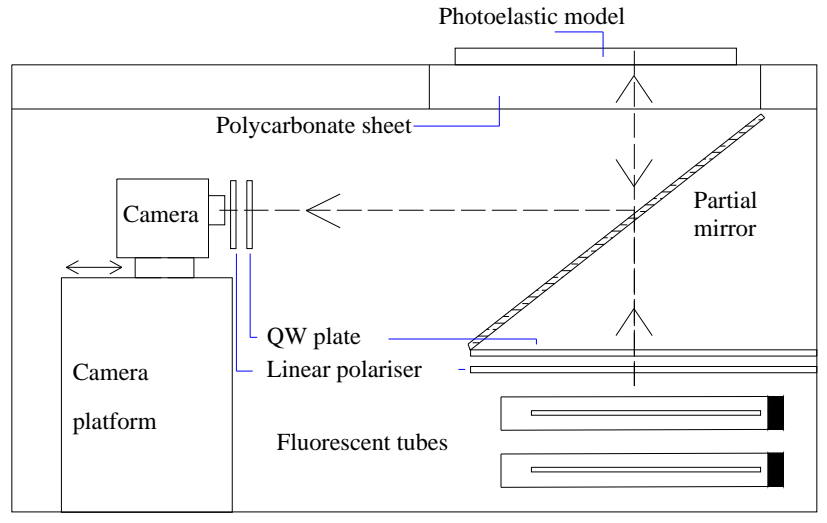

Figure 5. Sensor for phase shifting experiment with a partial mirror.

\section{RGB CALIBRATION METHOD}

The RGB calibration technique as discussed above was applied to determine the complete fringe order for fringe patterns obtained under the indenter load (Fig. 3a). The center of pattern was masked due to high stress region under the indenter head (theoretically the fringe order in this region is greater than three). Figure 6(a) shows the fringe patterns after masking with line $a-b$ along which the fringe order was determined as shown in Figure 6(b). 

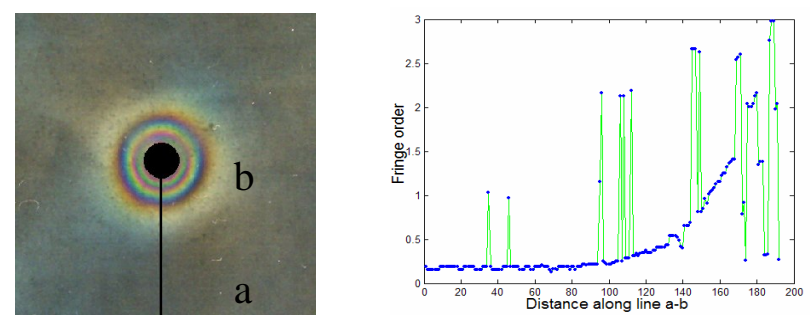

Figure 6. Fringes under unconventional loading (a) fringes due to indenter loading, (b) fringe order along line $a-b$.

From the fringe order principal stress difference can be obtained using equation (4) and stress analysis can be performed at every point along the line.

$$
\left(\sigma_{1}-\sigma_{2}\right)=\frac{N}{2 t} \frac{E}{(1+v)} \frac{\lambda}{K}
$$

where $N$ is the fringe order, $t$ is the material thickness, $E$ is the modulus of elasticity, $v$ is Poisson's ratio, $\lambda$ is the wavelength of light used and $K$ is the strain optic co-efficient of the material.

As can be seen in Fig. 6(b), with the conventional RGB method fringe order beyond 1.5 is difficult to track and had a limited accuracy in the available range. The main reasons for this are (i) due to method itself which allows maximum fringe order of three to be determined in addition to the type of light source and image acquisition system used (ii) the unconventional loading of surface deforms the material to a larger extent thereby the color chat of calibration LUT that can prepared through conventional loading does not truly represent the colors being induced under such deformations; this compromises the accuracy of the results obtained, (iii) the fringe pattern generated under surface loading through indenter are densely packed making the analysis difficult for fringe order determination. Thus this technique cannot be relied for sensing applications and requires some alternative approaches for accurate analysis.

\section{PHASE-SHIFTING METHOD}

In order to study the capabilities of phase shifting method and how far it can be implemented to achieve reasonable accuracy over the entire region this method was evaluated. Note that the technique was applied only to the fringes induced due to the vertical load, if the technique is useful for this load it can be equally applied to the fringes induced due to shear load. To determine whole-field fringe order for the entire region of interest (ROI), phase stepped images were acquired at six different optical arrangement of circular polariscope to fit into equations (2-3) to obtain the isoclinics and isochromatics. The central region of fringe pattern has high stress zone from the indenter head propagating along the perpendicular direction of the surface.

As explained earlier, the experimental setup for sensing application necessitated use of reflection photoelasticity with a partial mirror for arranging the light source and camera at two different orientations to avoid their interference. The experiments showed that images acquired under white light were of low contrast due to the presence of partial mirror in the design. Rotation of optical elements caused further glare and ghost images of the light source; significant loss in contrast was also observed due to glare from the white light source. However, the effect was found to be less prominent with the use of monochromatic light. Thus phasemap of isoclinics and isochromatics were obtained under monochromatic light using the equations (2-3) as shown in Figure 7. The isoclinics and isochromatics are influenced by model geometry, point of application of load, accuracy of axis orientation of filters and the relative retardation as a result of the applied load.
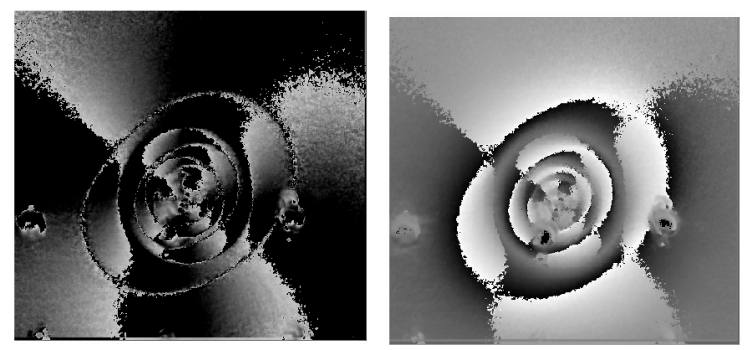

Figure 7. Phasemap using monochromatic light (a) isoclinics, (b) isochromatics

As can be seen in these figures the phasemap is oval rather than circular, this is due to the inaccuracy in orienting the filter axis and there are erroneous zones in the phasemap. The expected phasemap should have increasing fractional fringe order radially inwards but the phasemap obtained from images in the figure had reversed phasemap at some locations and erroneous output elsewhere. This has been also reported in the literature [1]. This was investigated and was found to be due to the ambiguity in determination of isoclinic as it fell in range of $-\pi / 4$ to $\pi / 4$, thus it could represent either $\sigma_{1}$ or $\sigma_{2}$ (principal stresses). It is important to note that isoclinic would represent only one of the two stresses to determine isochromatics and to produce accurate phasemap.

It has been found that Equation (2) is insensitive to fast or slow axis of the model as signs of numerator and denominator are canceling out, on the other hand Equation (3) is sensitive to the axis and the results can be erroneous and in certain zones the phasemap can appear to be reversed. This drawback was overcome by modifying the isochromatic equation (3) to (4); this equation is insensitive to fast or slow axis of model.

$$
\delta=\tan ^{-1}\left(\frac{ \pm \sqrt{\left(i_{5}-i_{3}\right)^{2}+\left(i_{4}-i_{6}\right)^{2}}}{\left(i_{1}-i_{2}\right)}\right)
$$

Figure 8(a) shows the phasemap obtained after applying equation (4) and Fig. 8(b) gives the fractional fringe order along line ' $a-b$ ' under monochromatic light source. The limitation of using equation (4) is that fringe order can only 
range between 0 to 0.5 if the positive square root is used or 0.5 to 1 if negative is used rather than 0 to 1 . Thus the advantage of identifying fringe order from equations (3) was lost, therefore to achieve the complete fringe order direction of fringe order is required.
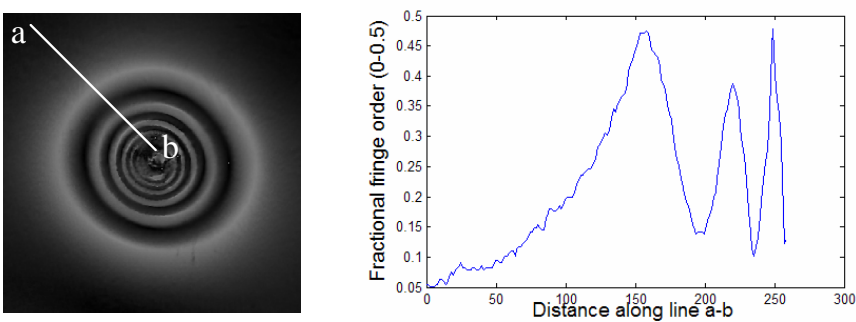

Figure 8. Phasemap under monochromatic light (a) with modified equation, (b) fractional fringe order along a-b.

For fringe patterns induced in these experiments the fringe order always increases radially from the edge of ROI towards the center, thus complete fringe order can be determined by phase unwrapping and can be substituted in equations (4) to obtain stress field for the entire model. A robust algorithm is required to unwrap the phasemap correctly in addition to the integral fringe orders and the trend of fractional fringe order of an image. The unwrapped phasemap for Figure 8 (a) is shown in Figure 9 with complete fringe order obtained along line 'cd'.
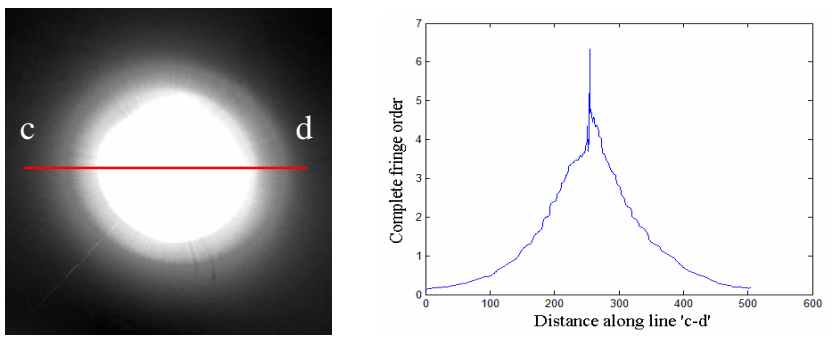

Figure 9. (a) Unwrapped phasemap with, (b) complete fringe order along 'c-d'.

Since the stress at the centre of the fringe patterns is too high and fractional fringe order obtained from phase shifting is unreliable and erroneous in this zone, the complete fringe order cannot be accurately unwrapped as can be seen in the adjacent line profile (Figure 9b).

\section{PHOTOELASTIC TECHNIQUES IN SENSING}

A case study on an actual foot image was carried out to evaluate the use of photoelastic techniques on complex fringe patterns and how far these techniques can be implemented in terms of quantitative measurements. Figure 10(a) shows portion of a foot print obtained under actual foot loading and Fig. 10(b) is the phasemap obtained using this technique.
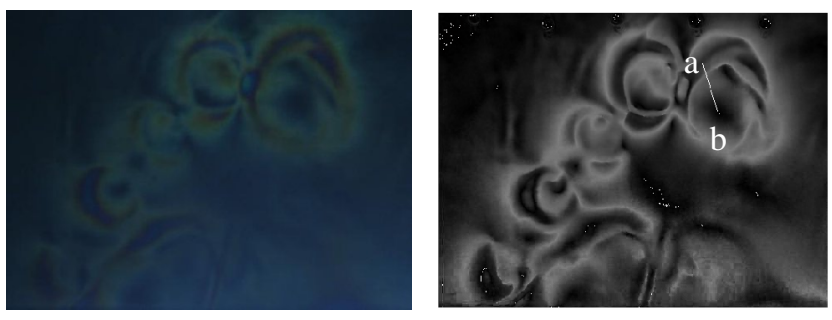

Figure 10. (a) Fringe patterns under foot loading (b) phasemap

The phasemap obtained here has the same limitations as the one obtained in Figure 8 and thus for determining the complete fringe order further processing would be required to integrate the entire fringe order. However, visual inspection of Figure 10(a) shows how complex and random fringe patterns appear to be under the actual foot loading compared to the patterns with controlled loading under the indenter. This makes it difficult to unwrap the phasemap and would necessitate manual efforts. Figure 11 shows the fractional and complete fringe order profile obtained along line ' $a-b$ ' from the above image. A complete fringe order for the entire image can be obtained, however, the process would be tedious and still inadequate to provide accurate information on the fringe patterns. When RGB calibration technique is applied to the foot image it would render exactly similar results as obtained for the indenter setup. However, it would be difficult to obtain complete stress field for the whole foot, also the results would not be accurate due to inconsistent mapping of the RGB values between the model and the specimen due to model deformation.
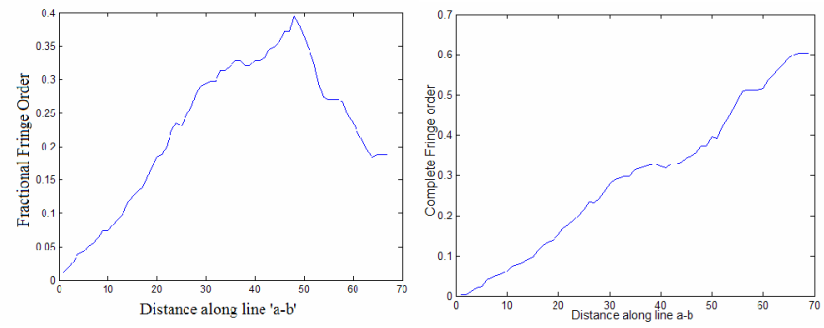

Figure 11. (a) Fractional and, (b) complete fringe order along line ' $a-b$ '

The results obtained show that conventional photoelastic techniques can only determine difference of principal stresses over a region of interest under unconventional loading conditions. For such loading situations as required in sensing, conventional photoelastic techniques have limited applications. Equation like Bousinesque [1] representing relation between principal stress difference and load are limited to a very few conventional setups and cannot be generalized, for instance it is only valid under the following conditions:

- Only for disc, thus other geometric shapes cannot be analyzed. 
- Only for diametral compression - if any additional load is applied at another point the equation is void.

- Invalid under high deformation of material.

However, for loading conditions where deformations of unknown shape may occur or repeatability of fringe patterns may not exist and have complex fringe patterns, mere determination of fringe order or stress difference is not sufficient for determining the complete stress field. Looking at the image from the actual foot loading (Fig. 10) it is evident that the image contains qualitative whole-field load information which can be extracted in a number of ways; (i) color of the image, (ii) geometry of the fringe patterns and, (iii) statistical parameter of the pattern from repeated loadings. However, these parameters cannot be combined in analytical forms using conventional photoelastic techniques. Hence, this would require some intelligent processing of the fringe patterns. Neural networks have been used as a model-free estimator to process photoelastic images in an idealized experimental condition that can precisely extract the load information from the images [16]. This, however, required a large number of images from identical loading conditions for training and tests. Whilst this may be the right approach in some applications it may not be a practical proposition for other applications; thus altogether a new strategy is required that can analyze complex images without requiring a large set of images. A knowledge-based adaptive system that can learn in real time and yet provide accurate results under varying test conditions may be the answer to this problem. Techniques like neuro-fuzzy and real-time data mining and forecasting models [17], which offer alternative approaches to such problems, may provide better options under these conditions.

\section{CONCLUSIONS}

In this paper design of a whole-field imaging sensor has been presented which can be developed for a number of sensing applications in biomedical and robotics areas. Use of conventional photoelastic techniques has been tested on fringe patterns obtained by surface loading of photoelastic material through a hemispherical indenter head. Both RGB calibration and phase shifting could only provide fringe order limited to the known region of stress. When these techniques were applied to obtain stress values for actual foot loading, it has been found that RGB method cannot be used to get the wholefield stress information whereas the phase shifting method is limited to obtaining stress field in controlled loading conditions due to the complexity of identifying the integral fringe orders from the irregular images. The results from the case study not only explain the limitations of conventional techniques but also identify the complexity of the problem. The results further show that although fringe patterns provide some qualitative data in terms of high stressed zones but determination of complete stress field was not possible with either of these techniques. This would require intelligent image processing techniques to be employed to extract the complete load information from photoelastic images and achieve the whole field analysis of the stress field.

\section{ACKNOWLEDGMENTS}

This research was supported by a bursary awarded by the School of Design, Engineering \& Computing, Bournemouth University.

\section{REFERENCES}

[1] Ramesh, K., 2000 "Digital photoelasticity: Advanced techniques and applications" Springer.

[2] Dubey, V. N. and Crowder, R. M., 2006 "A dynamic tactile sensor on photoelastic effect" Sensors and Actuators, 128: $217-224$

[3] Dario, P., Fermi, D. and Vivaldi, F., 1987 "Fiber-optic catheter-tip sensor based on photoelastic effect" Sensors and Actuators 12: 35-47.

[4] Jacobsen, S. C., Wood, J. E., Knutti, D. F. and Biggers, K. B., 1986 "The Utah/MIT dextrous hand: work in progress" in D.T. Pham and W.B. Heginbotham edited Robot Grippers, pp 341-389.

[5] Brooks, F., Ouh-Young, M., Batter, J. and Kilpatrick, P. 1990 "Project GROPE-haptic displays for scientific visualization" Computer Graphics, 24(4), 177-185.

[6] Eghtedari., F., Hopkins, S. and Pham, D., 1993 "Model of a slip sensor" Proc. of the IMechE Journal of Engineering Manufacture, 207, 55-64.

[7] Photoelastic Materials, Bulletin S-116-G, 1992, Vishay Measurement Groups.

[8] Grewal, G.S., Dubey, V.N., Claremont, D.J. (2006). Isochromatic demodulation by fringe scanning, Strain, Vol. 42, No. 4, pp. 273-281.

[9] Ajovalasit, A., Barone, S. and Petrucci, G., 1995 "Towards RGB Photoelasticity: Full-Field Started Photoelasticity in White Light" Experimental Mechanics, 35, 193-200.

[10] Quiroga, J., Botella, A. and Gomez-Pedrero, J., 2002 "Improved method for isochromatic demodulation by RGB calibration" Applied Optics, 41(17), 3461-3468.

[11] Ramesh, K. and Deshmukh, S., 1996 "Three fringe photoelasticity -use of colour image processing hardware to automate ordering of isochromatics" Strain, 32(3), 79-86.

[12] Patterson, E., 2002 "Digital Photoelasticity: Principles, Practice and Potential" Strain, 38, 27-39.

[13] Patterson, E., Hobbs, J. and Greene, R 2003 "A novel instrument for Transient Photoelasticity" Society for Experimental Mechanics, 43(4), 403-409.

[14] Asundi, A., 2002 "MATLAB for photomechanics: a primer" Elsevier.

[15] Quiroga, J., Servin, M., et al. 2002 "Regularization phase tracking technique for demodulation of isochromatics from a single tricolor image." Measurement Science Technology 13: 132-140. 
[16] Dubey, V. N., Grewal, G. S. and Claremont, D. J. 2006 "Extraction of Load Information from Photoelastic Images using Neural Networks" ASME Design Engineering Technical Conference, DETC2006-99067, Philadelphia, USA.

[17] Gabrys, B. 2002 "Neuro-Fuzzy Approach to Processing Inputs with Missing Values in Pattern Recognition Problems" International Journal of Approximate Reasoning, vol. 30, no. 3, pp. 149-179. 